\title{
Propelling Machinery for Naval Vessels
}

\section{Review of Present Conditions and Future Possibilities in Motive Power}

\section{By Rear-Admiral H. I. Cone, U. S. N, Engineer-in-Chief and Chief of Bureau of Steam Engineering}

THE advent of the turbine has greatly extended the continuously propelled by steam machinery. It is therefore, particularly suited to high speed vessels.

therefore, particularly suited to high speed vessels.
In the older destroyers a sustained speed of 30 knots necessitated driving the reciprocating engine at or beyond its limit of safety and reliability. As a result the contract speed could rarely be maintained on service, and certainly not for any extended period of time.

To-day the steam turbines of our destroyers can be driven continuously at full power as readily as at the lower powers.

Next below the destroyer in the speed scale is the scout cruiser; of these we have three, the "Birming. ham," "Chester" and "Salem," similar in design, except as regards their machinery installations. The "Birmingham" has reciprocating engines, the "Ches"Birmingham" has reciprocating engines, the "Chesbines. The contract trial speeds were, "Birmingham," 24.325 knots; "Chester," 26.52 knots; and "Salem," 25.947 knots, thus demonstrating, as in the case of the destroyers, the superiority of the turbine for development of high speed.

A rather unexpected result of our experience with these vessels on service has been the greater reliability of the reciprocating engine. In the case of this type of vessel, where the reciprocating engine is ad mittedly out of place, it was confidently expected that the turbine would demonstrate its superiority in this regard. Thus far, however, under similar conditions of service, the machinery of the "Birmingham" has required considerably less repairs than that of either turbine vessel, and of the three, the "Birmingham" has always been the one whose availability for service could most be relied upon.

Thus for the scont cruiser, while the turbine is the most suitable type of machinery, its superiority over the reciprocating engine is not so manifest as in the case of the faster destroyer.

As we descend the speed scale into the region of the more important naval vessels of medium speed, the turbine is still less clearly indicated as the best type of propelling engine. This is because of the relatively increased importance of its principal deficiencies which are:

(1) A tendency to disablement to a degree requiring repairs beyond the capacity of the ship's force, thus temporarily destroying the military value of the vessel.

(2) A lack of economy of steam consumption at the lower speeds, which affects the cruising radius of the vessel.

Regarding the first of these, there is a tendency to a rubbing and stripping of the turbine blading, due to the small. clearances necessarily employed, the causes of which are sometimes obscure, and the pre. vention of which is accordingly difficult.

The re-blading of a damaged turbine can be done only at a navy yard, and requires the lifting of the casing and the rotor, which in itself is a considerable item. In our experience this work has seldom been completed within a month.

Improvement in design, greater experience with the operation of the turbine, and better facilities for its repair, will doubtless reduce somewhat the extent and importance of this trouble. But at present the reliability of the turbine in service must be considered as probably not as good in a medium or low-speed vessel as that of the reciprocating engine in its present development, with forced lubrication of its bear. ings.

Inferiority of the turbine in its economy at the lower speeds is probably inherent. At the cruising speed of a battleship, the reciprocating engine is 20 to 30 per cent more efficient than the turbine.

These failings of the turbine are due in a great degree to the conditions governing its use in nava! vessels, and are not manifest in its other applications. When driven continuously at high-speed, as with electric generators, and, to a less degree, in highspeed passenger vessels, its efficiency as compared with that of the reciprocating engine is entirely satisfactory, and in a vessel the nature of whose service is such that the turbines can be kept warm during the short intervals between runs, the danger of stripping during the warming-up period largely disappears. The irregular movements of naval vessels, however, with their frequent periods in port with cold turbines and their considerable cruising at low speeds, impose handicaps on the turbine in these two respects.

The peripheral speed of turbines for maximum efficiency should be not less than half the velocity of the steam. To secure this maximum efficiency within practicable limits of diameter of the turbine requires, ordinarily, revolutions per minute of from 1,500 to 3,000 . These speeds are perfectly practicable for electric generators, but are unsuited to the marine pro peller, whose efficient working requires revolutions, depending upon its diameter, of from 70 to 800 pe minute.

There is presented, therefore, the problem of providing a method in which a high-speed turbine may be made to drive a slowly-revolving propeller, thus conserving both turbine and propeller efficiencies.

Two methods of accomplishing this are particularly prominent just now.

\section{Reduction-Gear Drive.}

The collier "Neptune," built by the Maryland Steel Company at Sparrow's Point, Maryland, has been equipped with reduction-gear machinery constructed by the Westinghouse Machine Company. This collier is a twin-screw vessel, displacing 19,300 tons, with a

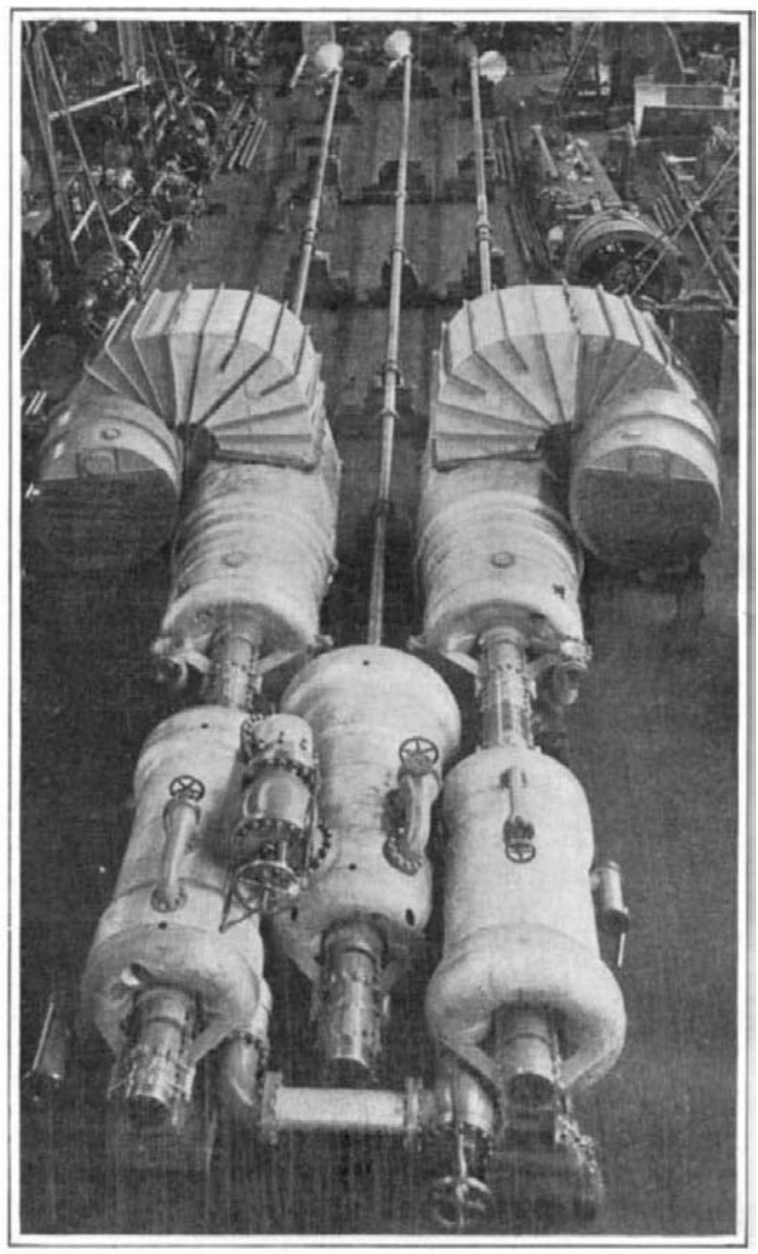

The turbines, shafting and propellers of a triplescrew destroyer, assembled in the shops.

speed of 14 knots. Steam at a pressure of 200 pounds is supplied to a Westinghouse Parsons turbin for each shaft, each turbine developing about 3,600 shaft horse-power on 1,220 revolutions. Between th turbine and its propeller shaft is mechanical gearing which will reduce the propeller speed to 135 revolutions per minute at about 14 knots. The gear pinions are carried in fioating frames supported by oil piston in such manner as to effectually take up any irregularity in the gear cutting. The oil pressure on pistons is indicated on gages which afford a means of directly reading the tooth pressure, and thus calculating the power being developed. A novel feature of the installation is a provision for operating the propelling machinery from the bridge. The shop tests of the gearing for the "Neptune" have been very satisfactory. The gears have demonstrated an efficiency of transmission in excess of 98 per cent, and have run quietly for long periods with no apparent wear. There is no back lash on reversing.

Extensive trials of the "Neptune" will be held

Reduction gearing as installed in the cargo steame "Vespasian" by the Parsons Company has successfully completed a year's trial, during which its durabilit has been tested under the severe conditions of weather in the North Sea during the winter months. The vessel has made 32 trips, a total of about 20,000 miles, carrying coal from the Tyne to the Continen and returning with water ballast. It is reported that during this time there has been no appreciable wea in the teeth of the gearing, and that there is practi- cally no slackness apparent, although no provision was made of adjustment for wear. A noticeable feature of the installation has been the almost entire suppression of the tendency to racing of the engine, even in weather when the propeller has been entirely out of water.

Electric Propulsion in a Naval Collier.

In the naval collier "Jupiter," a vessel similar to the "Neptune," which is being constructed at the navy yard, Mare Island, California, there will be obtained a trial of a method of electric propulsion. A contract for this machinery has been placed with the General Electric Company. In this system it is proposed to in stall one turbo-generator running at a maximum speed of about 2,000 revolutions per minute, and delivering current with a potential of 2,300 volts to induction motors, one on each propeller shaft. The power from the turbine will be transmitted to the propeller shafts, electrically, with a speed reduction in ratio of 18 to 1 . The efficiency of transmission is expected to be about 91 per cent. The water rate for this installation is stated to range from 12.15 pounds per shaft horse power at 14 knots, to 15.55 pounds at 10 knots.

In the case of a battleship the proposed form of electric drive contemplates two turbo generators driving two motors on each of the two main shafts. This multiplicity of units combined with a pole-chang. ing device on two of the motors, promises an economy close to the maximum at all speeds above 10 knots.

Comparing the electric with the mechanical reduction-gear it is at once evident that the latter has the superior efficiency of transmission. In the case, however, of a vessel in which economy at the lower speeds is required, the flexibilty of the electric in stallaticn, as outlined for a battleship, might overcome this disadvantage.

As with the scout cruisers, we will, therefore, probably have in service three similar colliers with different types of propelling machinery, the "Cyclops" being already in commission with an excellent installation of reciprocating engines, which on trial déveloped a horse-power on less than 1.5 pounds of coal per hour at full power.

Combination of Turbines with Reciprocating Engines.

The combination of a reciprocating engine working the steam from boiler pressure down to nearly atmospheric pressure, and a turbine which works from the exhaust pressure of the reciprocating engine down to the vacium, is a particularly efficient one, because under such conditions each engine is working in the pressure range to which it is best suited. Such combinations are used with great success in power plants on shore and in certain moderate-speed passenger vessels, notably the "Olympic," "Titanic," "Laurentic," and "Otaki." Its principal value is in economy of steam consumption at full power in a medium speed vessel, in which case it probably gives a gain in economy over the reciprocating engine or turbine of not less than 10 per cent. In the case of naval vessels whose cruising speed requires only from $1 / 7$ to $1 / 12$ of full power, the advantage of this combination is of full power, th
not so impressive.

There is, however, a combination of reciprocating engine and turbine which has its maximum efficiency at the cruising speed. This consists in the use of a small reciprocating engine in a manner similar to that of the cruising turbine in a Parsons turbine installation. The reciprocating engine, whose size is limited to that required to pass the steam for the designeil cruising speed, exhausts through the turbines. At higher speeds the reciprocating engine is disconnected. The weight of the reciprocating engine, and the space required, are less than for the cruising iturbines, and it is probable that the engine will be less proné to disablement than the cruising turbine. The principal difficulty involved seems to be the design of the clutch connecting the reciprocating engine. with the turbine shaft.

In another combination which suggests itself, and which, theoretically, has about the same efficiency, there is substituted for the reciprocating cruising element a small high-speed turbine connected by reduction gearing to the main turbine shaft.

The selection of machinery for a naval vessel at the present time, therefore, requires a careful consideration of a number of possible types. The line drawings on page 519 show the approximate appearances of systems which have been considered for a battleship

\section{Heavy-Oil Engine.}

It now seems probable that none of the foregoing methods of propelling medium-speed naval vessels, 
all of which are forms of steam machinery, will endure. This in consequence of the recent remarkable development of in Europe. Hitherto, oil engines have not merited much consideration for large naval vessels on account of the limited power that could be developed in a single cylinder. An installation of any considerable power requiıed a multiplicity of cylinders. Now, however, we are credibly informed that 1,000 horsepower has been developed in a cylinder about 33 inches in diameter with a in-inch stroke, at 150 lutions per minute, in a 2-cycle marine type readily reversible engine. This engine has a speed con trol that is satisfactory, and an economy of fue consumption probably twice that of a steam en gine. An experimental double-acting marine en gine, with three cylinders, of about this same size, has been built to develop 6,000 brake horse-power at 150 revolutions per min ute. In the development of the oil engine, the trunk piston is being abantoned and cross-heads and connecting rods used. It will, therefore, resemble the reciprocating steam en gine, and our experience with the latter will prepare us for the operating and repairing of the oil engine.

As in the case of all first installations of whatever type, there will probably be disappointments in the first applications of the oil engine in marine propul sion, and it may be that further development of the engine may be found necessary prior to its extensive adoption. However, the superior economy of the oil engine, together with the elimination of the steam boiler and condenser will cause us to be patient in the face of a considerable amount of trouble. The extent of the alop tion of the oil engine by the nations of the world will be largely affected by the supply of oil available. We are in a particularly fortunate position in this respect, producing as we do two-thirds of the world's supply.

\section{Use of Fuel Oil in Place of Coal,}

In our new construction we are greatly extendin the use of oil in steam vessels. All our new destroythe use of oil in steam vessels. All our new destroy
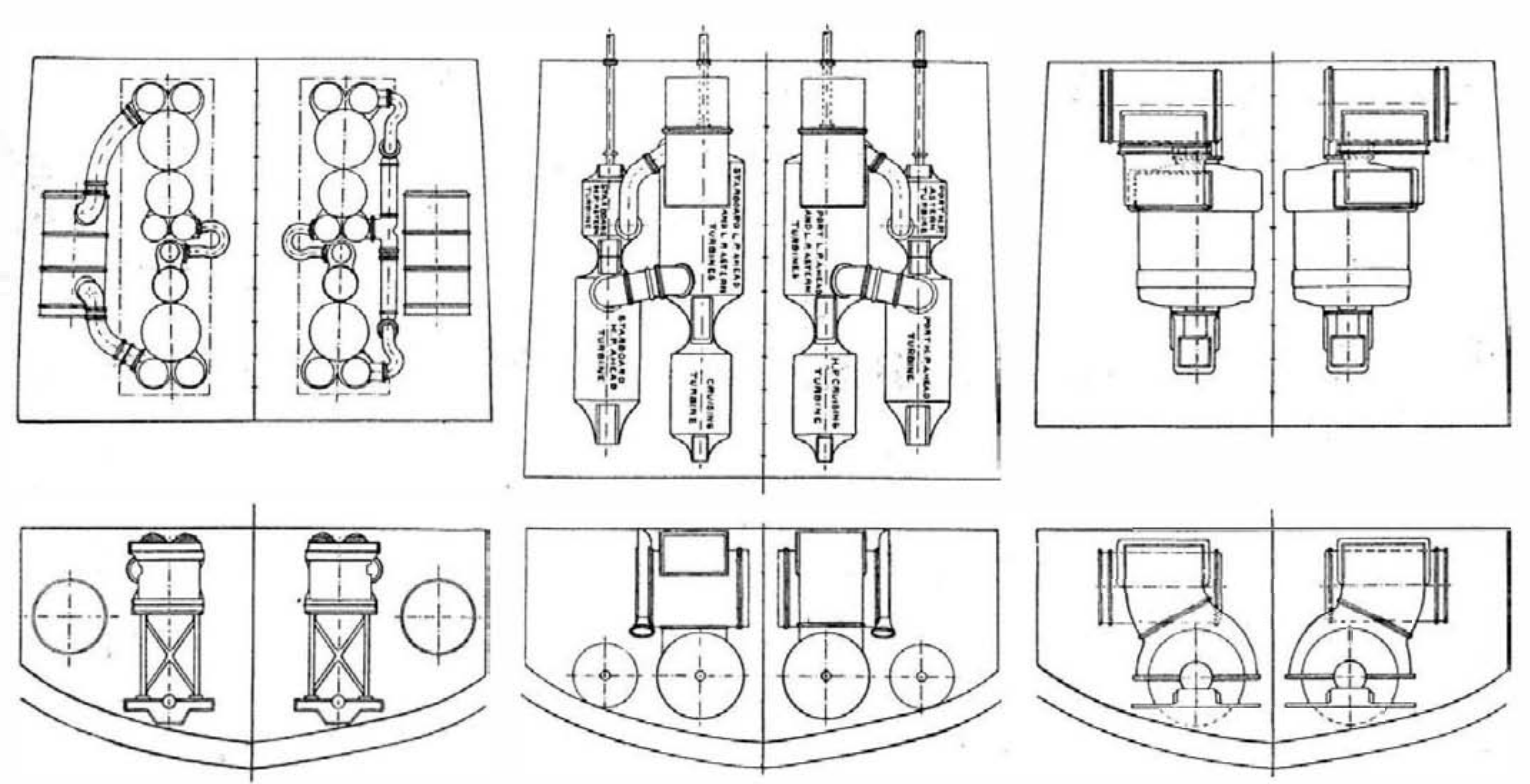

Reciprocating engines.

Parsons turbines.

Curtis turbines.
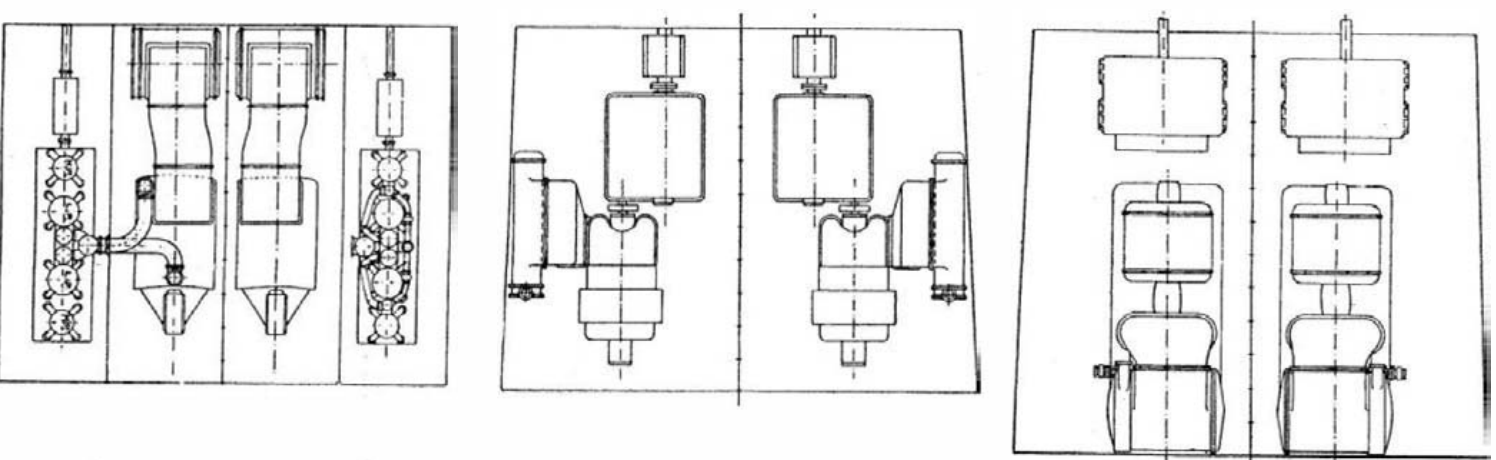

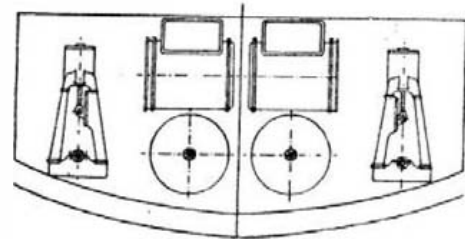

Combination of reciprocating engines and turbinies.

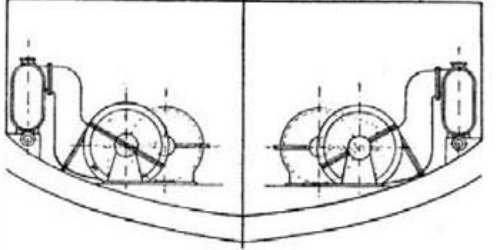

Turbines with mechanica reduction gear.

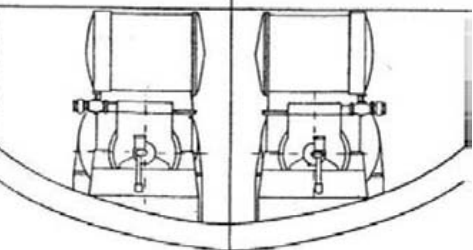

Turbines with electric motor propulsion.
ENGINE ROOMS OF SAME SIZE AS "DELAWARE'S" (RECIPROCATING EQUIPPED WITH YARIOUS TYPES OF PROPELUING MACHINERY
- the larger vessels, including battleshins some of flows: than with coal in the ratio of about 14 to 9 , and per quare foot of heating surface in about the ratio of to 8 , is obtained. Fuel oil can be taken aboard the routine of the ship. Steam for full power in such a vessel can be maintained as readily as for low power. A vessel burning oil is capable of prolonged runs at full speed limited in length only by the supply of fuel. There is no reduction in speed due to dirty fires or to difficulty in trimming coal from remote bunkers.

There are no cinders and the amount of smoke can be controlled.

A considerable reduction in personnel is possible, the size of the fireroom force required being limited to that necessary for overhaul and cleaning of the plant.

The weight and space required for boilers is reduced, first, by the reduction in heating surface $\mathrm{rc}$ quired, and second, by the shortening of firerooms, no space being required in front of boilers for handling fire tools.

Coal and ash handling gear is eliminated. This renders unnecessary the piercing of the hull for coal trunks and for discharges from the ash expellers or ash ejectors.

The stowage and handling of oil is much easier than of coal, and will result in a much cleaner ship and consequent increase in time available for drills. The mechanical supply of fuel to the boilers gives a prompt and delicate control of the steam supply, permitting more sudden changes in speed than with coal, which is a tactical advantage.

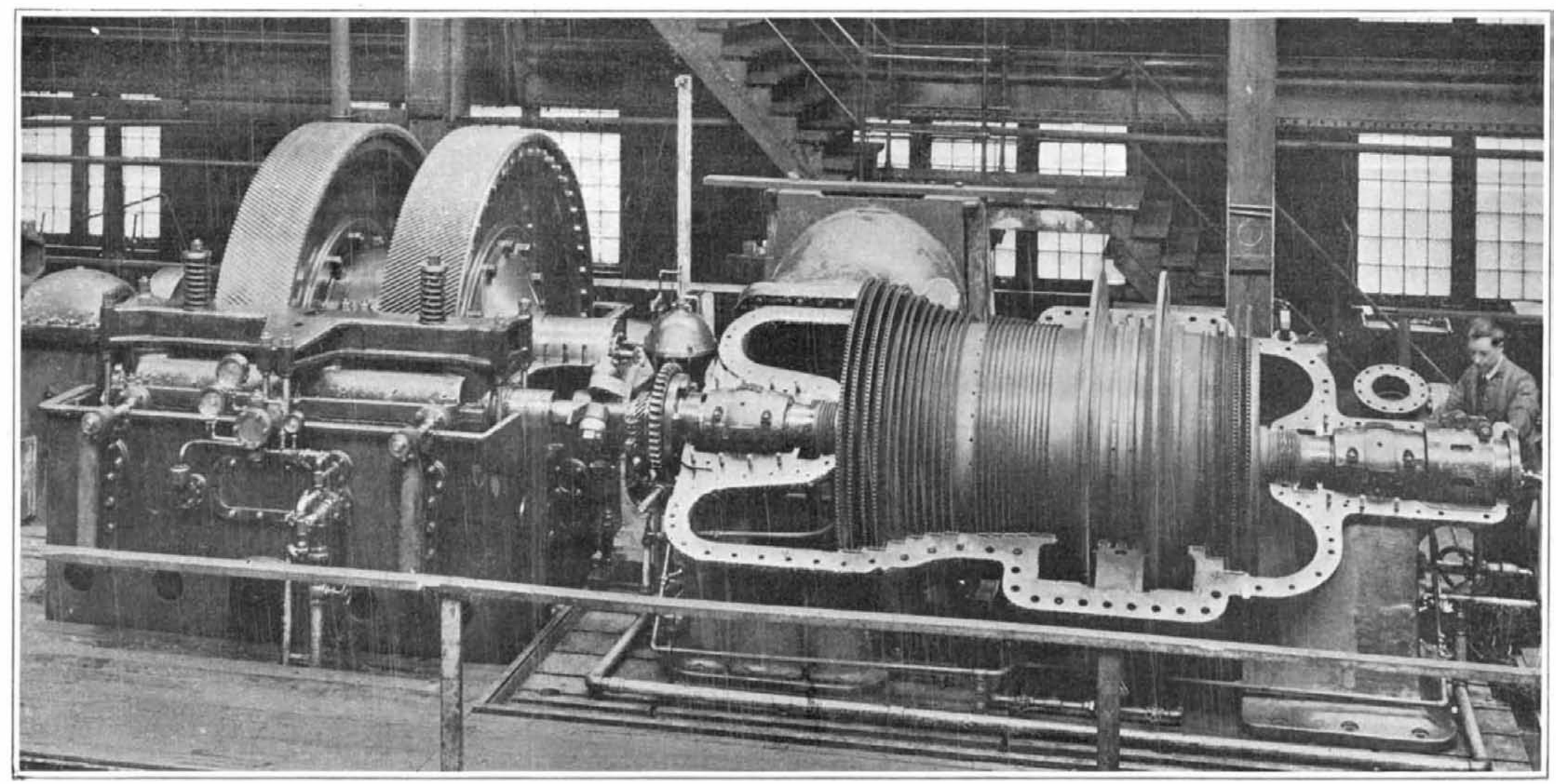

Speed of turbine, 1,220 revs. per min. Speed of propellers, 135 revs. per min. Efficiency of reduction gear, 98 per cent. 